Journal of the Egyptian Society of Parasitology, Vol.43, No.2, August 2013 J. Egypt. Soc. Parasitol., 43(2), 2013: $471-480$

\title{
LIMB-SALVAGE ANGIOPLASTY IN POOR SURGICAL CHRONIC LIVER DISEASE AND DIABETIC PATIENTS
}

By

HUSSAM HAMDY ${ }^{1}$, M. EL-KOLLY ${ }^{1}$, H. EZZAT ${ }^{2}$, M. ABBAS ${ }^{2}$,

Y. FAROUK ${ }^{2}$, M. NASER ${ }^{2}$, M. MAGDY ${ }^{2}$ and A. ELRAOUF ${ }^{2}$

Theodor Bilharz Research Institute ${ }^{1}$, Embaba 30, Giza and National

Institute of Diabetes and Endocrinology ${ }^{2}$ (NIDE), Egypt

\section{Abstract}

Critical limb ischemia (CLI) in high surgical risk patients with chronic liver diseases has a grave prognosis with a one-year mortality rate of $20 \%$ and a oneyear amputation rate of $25 \%$ after the initial diagnosis. According to Trans-Atlantic Inter-Society Consensus (TASC)-II Guidelines, revascularization (surgical \& endovascular) is the treatment of choice for patients with critical limb ischemia (CLI). The primary goal of revascularization is to relieve ischemic rest pain, heal ulcers, prevent amputation, improve patient's quality of life (limb salvage) and secondary goal was the periprocedural complications. Endovascular techniques include balloon angioplasty, stents, stent-grafts, and plaque debulking procedures. Surgical options, identification of patients at risk of postoperative complications could have an impact on the indications for a procedure as well as permitting modifications of treatment to reduce the surgical risk.

This study evaluated the treatment out comes after limb salvage angioplasty for patients who otherwise would be candidates for primary amputation due to poor co-morbid conditions as chronic liver disease and diabetes.

The clinical evaluation, laboratory investigations and abdominal ultrasonography were performed to all patients to evaluate their liver status. Patients were classified according to Child-pugh classification into child A, B \& C. All patients were subjected to either detailed arterial duplex or C.T. angiography to assess their arterial lesions from January 2008- January 2010. 95 patients with critical limb ischemia (Rutherford categories $4,5,6$ ) were treated by primary percutaneous transluminal angioplasty (PTA). No patient was excluded on the basis of the extent of arterial occlusive disease. The primary end points were immediate technical success, clinical improvement and limb salvages rates. Secondary end points were periprocedural complications and mortality.

Most of the patients were male (54.7\%) with mean age 62 (48-70 years). Underlying cirrhosis due to $\mathrm{HCV}$ was $(82.2 \%)$, HBV (5.4\%), while mixed viral infections was (12.4\%). 54\% were categorized as Child B, $32 \%$ as child A and $14 \%$ as child C. Associated diabetes mellitus was present in $96 \%$ of the cases, hypertension in $64.2 \%$, ischemic heart disease in $74 \%$ and hyperlipedemia in $32 \%$. Rest pain, tissue loss, or both, were the presenting symptoms in $83 \%$ while infec- 
tion and ulcer were present in the other $17 \%$ of patients. The total numbers of interventions were 154; the treated lesions were 89 in the tibial arteries, 12 in the popliteal artery, 44 in the superficial femoral artery, 3 in the common femoral artery and 6 in the iliac arteries with initial technical success rate of $93.6 \%$ and periprocedural complications of $12.6 \%$. All patients were in Rutherford clinical category 4, 5, 6 none of these patients had a previous bypass operation. Mean follow-up was 15 months. The limb-salvage rate was $87.4 \%$. Eighty patients (84.2\%) of toe amputation sites healed primarily. three patients with rest pain had resolution of their symptoms after angioplasty. All technical failures were due to inability to cross the lesions. Of the 6 technical failures, 4 required amputation, and 2 refused any further therapy.

Key wards: Percutaneous Trans-luminal Angioplasty (PTA), Critical lower limb ischemia, Postoperative complications, Diabetes, Chronic Liver Disease.

Correspondence: Dr. H. Hamdy, Email: hamdy.hussam@gmail.com

\section{Introduction}

The chronic critical lower limb ischemia occurs in the setting of severe peri-pheral arterial disease that is often characterized by advanced atherosclerosis at multiple level lesions in the lower extremity (Arora et al, 2013). Despite the challenges posed by complexity of arterial disease in such patients, endovascular intervention is a less invasive alternative to bypass graft surgery in most patients, with low procedural morbidity and mortality. Continual advances in percutaneous techniques have made it possible to revascularize lesions considered nonamenable for endovascular intervention. The development of dedicated peripheral wires, balloons, stents, and catheters allows recanalization and revascularization of almost any anatomy (Hiatt $e t$ al, 2007). Patients commonly presenting with critical limb ischaemia (CLI) are often elderly with multiple comorbid conditions (Park et al, 2007). Patients were considered poor bypass operation candidates on the basis of the absence of an adequate greater saphenous vein for distal bypass, the presence of a poor distal bypass target vessel, or the presence of sever comorbid conditions.(Chronic liver disease, uncontrolled diabetes, recent myocardial infarction $<6$ weeks, symptomatic coronary artery disease, or sever chronic obstructive pulmonary disease). Hepatitis $\mathrm{C}$ virus (HCV) infection is a major worldwide health problem that has an increasing prevalence and mortality. $\mathrm{HCV}$ is the most frequent cause of chronic liver disease and $20-30 \%$ of patients develop cirrhosis with a risk of hepatocellular carcinoma (El-Zayadi et al, 2005). It is estimated that 360 million suffer from chronic HBV infection worldwide. Both HBV \& HCV are transmitted parentally and coinfection is not uncommon in intravenous drug users and in countries with a high prevalence of $\mathrm{HBV}$, also may result in more severe liver disease than either infection alone (Ezzat et al, 2005). Also, the chronic liver disease association with diabetes mellitus may be attributed to common causes e.g. 
alcoholism, hemochromatosis, and the possibly autoimmune conditions. Viral hepatitis (HBV \& $\mathrm{HCV}$ ) is established causes of chronic liver disease and seems to occur more frequently in diabetics than in the general population (Schreuder et al, 2008). This association may reflect epidemiological circumstances because of the frequent parenteral exposure of diabetics (Liao et al, 2009).

The purpose of this study was to evaluate the treatment out comes after limb salvage angioplasty in high surgical risk patients with chronic liver diseases, otherwise for primary amputation.

\section{Patients, Materials and Methods}

A retrospective review of management of 95 patients with critical limb ischemia was classified according to Rutherford into the categories 4, 5, 6 (Rutherford, 2010), in association with chronic liver disease and diabetes were admitted to Theodor Bilharz Research Institute, National Institute of Diabetes and Endocrinology (NIDE), El-Gomhorya Hospital and El-Haram Hospital from January 2008- January 2010 treated by the primary percutaneous transluminal angioplasty (PTA). No patient was excluded on the basis of the extent of arterial occlusive disease. The primary end points were immediate technical success, clinical improvement and limb salvages rates. Secondary end points were periprocedural complications and mortality. All patients' demographic data including full history taking and clinical evaluation to assess the liver status, diabetes, cardiological condition and other risk factors. History of previous attack of bleeding (hematemesis or melena), cigarette smoking or myocardial ischemia was recorded.

Routine laboratory investigations including: complete blood picture, liver function tests, renal functions tests, total proteins, serum albumin, coagulation profile, hepatitis markers and fasting \& postprandial blood sugar were done. Abdominal ultrasonography examination was performed to all patients. Diagnosis of liver cirrhosis was based upon sonographic findings of dilated portal vein, increased liver echogenicity, splenomegaly and/or ascites, upper endoscopy (gastroscopy) to verify of esophageal varices and its degree. Patients were classified according to Child-pugh classification into child A, B \& C were collected and tabulated, clinical criteria including Rutherford category at baseline and during follow up were collected and analyzed. A Peri-procedural medication was acetylcysetine with good hydration at a rate of $0.5 \mathrm{ml} / \mathrm{Kg} / \mathrm{hr}$ normal saline for $6 \mathrm{hrs}$ before and after the procedure, intra-procedural 5000 units of unfractionated heparin.

The non-ionic contrast medium (ultravist) was used Post procedure, therapeutic low molecular weight heparin (LMWH) anticoagulation for $48 \mathrm{hrs}$. together with clopidogril loading dose (300mg), then statins (Atorvastatin; $20 \mathrm{mg}$ /day) and clopidogril (75mg/day) were continued on maintenance dose for 90 days and subsequently acetyl salicylic acid $100 \mathrm{mg} /$ day or ticlopidine $250 \mathrm{mg}$ /day indefinitely. The procedure was performed under local anesthesia through an antegrade approach of the 
ipsilateral common femoral artery, or through a contralateral approach if obstruction of both iliac and common femoral artery. The trans-brachial and trans-popliteal approach was also used in patients with bilateral iliac artery occlusions. Procedural tools were invariably, a six French gauge sheath positioned to perform a preliminary angiographic study using diluted (50\%) non-ionic contrast medium. 0.0140.035 inch hydrophilic guide wire together with a diagnostic catheter (4-5 French) to locate the lesion and to support the wire and 3.0-5.0 French balloon catheter were used for the dilatation of 2-8 $\mathrm{mm}$ diameter arteries. The authors tried to avoid the use of stents especially in the infrapopliteal arteries since the risk of thrombosis is very high in such low-flow vessels. Stents were employed if there is subintimal dissection. Post-procedure, any minor amputations needed were to be done within $48 \mathrm{hrs}$. of the procedure, together with recording of periprocedural

adverse events. Follow up was scheduled at 1 month, 3 months, 6 months, and 1 year following the procedure for: (1) sustained clinical improvement based on absence of rest pain and/or progressive tissue healing; (2) Freedom from major amputation. All the data regarding patients who were not followed directly by the present authors were obtained from colleagues treating these patients.

\section{Results}

Ninety-five patients underwent percutaneous trans-luminal angioplasty (PTA) for critical limb ischemia (CLI) during our study period; we studied 52 males and 43 females, with a mean age of 62 years, associated with chronic liver disease. Diabetes $(96 \%)$, hypertension $(64.2 \%)$, ischemic heart disease (74\%), smoking (32\%), hyperlipedemia $(32 \%)$ and cardiovascular stroke $(16 \%)$ are associated risk factors.

Table1: Patients' risk factors:

\begin{tabular}{|l|l|l|l|}
\hline & No. patient & No. patient & No. patient \\
\hline Underlying Cirrhosis & HCV 78 (82.1\%) & HBV 5 (5.3\%) & Mixed 12 (12.6\%) \\
\hline Child Pugh Classif. & Child A 31 (32.6\%) & Child B 51 (53.6\%) & Child C 12 (12.6\%) \\
\hline \multirow{2}{*}{ Associated risk factors: } & 1-Diabetis 91 (96\%) & 2-IHD 70 (74\%) & 3- Smoking 30 (32\%) \\
\cline { 2 - 4 } & 4- HTN 61 (64.2\%) & 5- Hlipid. 30 (32\%) & 6- CVS 15 (16\%) \\
\hline
\end{tabular}

The clinical stratification of our patients at time of enrolment in our study showed unequal distribution between Rutherford categories 4, 5, 6. None of these patients had had a previous bypass operation.

Table 2: Clinical presentations

\begin{tabular}{|l|c|c|}
\hline Clinical Presentations & No. Patients & Percentage \\
\hline Gangrene & 7 & $7.4 \%$ \\
\hline Ulcer & 11 & $12 \%$ \\
\hline Infection & 5 & $5 \%$ \\
\hline Rest Pain & 4 & $4 \%$ \\
\hline
\end{tabular}

Lesions were classified in the treated limbs according to the target vessel to be treated which is the most likely to provide an in line flow down to the 
foot. This was done during the intraprocedural preliminary selective contrast injection together with digital subtraction angiography (DSA) for proper visualization of the vessels and careful differentiation between a tight stenosis and total occlusion. In case of the presence of a simultaneous stenotic and occlusive lesions, or 2 lesions of the same type but with varying lengths in the same target vessel, we classified the lesion according to the level of the lesion into one level lesion angioplasty (40 limbs), two level lesions (51 limbs) and three level lesions (4 limbs). Tibial vessels were the most commonly treated with a $57 \%$ preponderance, the superficial femoral artery was our target vessel in $29 \%$ of treated limbs, the popliteal artery (8\%) while common femoral and iliac arteries where $2 \%, 4 \%$ respectively.

Table 3: Anatomical arterial lesions according to child-Pugh classification

\begin{tabular}{|l|c|c|c|c|c|}
\hline Intervention & $\mathrm{N}=154$ & percentage & Child A & Child B & Child C \\
\hline Tibial a. & 89 & $57 \%$ & 18 & 34 & 7 \\
\hline Popliteal a. & 12 & $8 \%$ & 4 & 6 & \\
\hline SFA & 44 & $29 \%$ & 5 & 10 & 1 \\
\hline CFA & 3 & $2 \%$ & 1 & 3 & \\
\hline Iliac a. & 6 & $4 \%$ & - & 5 & 1 \\
\hline
\end{tabular}

The technical characteristics of the procedure regarding guide-wire passage plane through the lesion were determined by the operator according to the guide-wire lesion interaction under fluoroscopic guidance, and were documented in the procedure details sheet.
Of 95 approaches 73 had $(76.8 \%)$ limbs through ipsilateral approach, 20 (21.1\%) limbs through a contralateral approach, $1(1.1 \%)$ limb through transbrachial, transpopliteal approaches respectively.

Table 4: Procedure technical approach

\begin{tabular}{|l|l|l|}
\hline Ipsilateral & 73 & $77 \%$ \\
\hline Contralat. & 20 & $21.1 \%$ \\
\hline Brachial & 1 & $1.1 \%$ \\
\hline Popliteal & 1 & $1.1 \%$ \\
\hline
\end{tabular}

Immediate technical achieved success, which was defined as residual angiographic stenosis of $<30 \%$ of the target lesion, in $89(93.6 \%)$ of our procedures with only 6 failures in 6 patients of those, 4 underwent major amputation and 2 patients refused further intervention and the reasons for failures were inability to cross a highly calcified chronic total occlusion. these patients were not suitable for surgical revascularization and had primary be- low knee amputations. Limb-salvage rate was $87.4 \%$. The eighty patients $(84.2 \%)$ of toe amputation sites healed primarily, three patients with rest pain had resolution of their symptoms after angioplasty. There were 8 major amputations, limb loss occurred in four patients after technical failure and in four others, despite a technically successful angioplasty, limb loss was due to infection in four patients despite initial clinical improvement. Three patients died 
due to myocardial infarction and one discontinued.

Table 5: Outcome, peri-procedural adverse events and mortality

\begin{tabular}{|l|c|c|}
\hline Outcome & No. Patients & Percentage \\
\hline Healing or minor amp. & 80 & $84.2 \%$ \\
\hline Rest Pain Improvement & 3 & $3.2 \%$ \\
\hline Major Amputation & 8 & $8.4 \%$ \\
\hline Discontinued & 1 & $1.1 \%$ \\
\hline Death & 3 & $3.2 \%$ \\
\hline
\end{tabular}

During follow up, the recurrence of clinical symptoms correlated with recurrent arterial stenosis in 12 patients. Of the 12 patients in whom clinical restenosis occurred, PTA procedures were successfully repeated in 10 patients. In the other 2 patients (child $\mathrm{C}$ group), a further PTA was not feasible. All recurrence and clinical failures led to major amputation within 6 months of primary angioplasty.

\section{Discussion}

Chronic liver disease patients' requiring surgery were at greater risk for surgical and anesthetic related complications than those with a healthy liver. The risk magnitude depends upon the type of liver disease and its severity, the surgical procedure, and the type of anesthesia (Pandey et al, 2012). To quantify the surgical risk in vascular patients, there are three principle types of risk that must be assessed-the risk to the patient from the primary vascular disease, the risk to the patient from concomitant medical problems and the risks that each available treatment option holds (Patel et al, 2011). Multiple factors contribute to the increased risks that patients with liver disease have when undergoing surgery. Accurate preoperative identification of patients with liver disease allows their treatment plans to be adjusted accord- ing to severity and nature of the underlying disease, as well as the type of surgery being undertaken. In patients with acute liver disease, elective surgery should be postponed until symptoms resolve. In patients with chronic liver disease, peri-operative risk increases with worsening severity of hepatic dysfunction as assessed by either the Child classification or MELD score (Davies and Ranaboldo, 1994). The preoperative management of complications related to patients' under-lying liver disease was essential to optimize their outcomes. Following surgery, patients with liver disease should be observed closely for the hepatic decompensating that often presented with worsening jaundice, encephalopathy, and ascites (Mohan et al, 2007). The best biochemical measures of liver function are probably the prothrombin time, serum albumin and serum bilirubin concentration. However, the serum bilirubin concentration usually rises, particularly after complicated surgery, multiple blood transfusions, excessive bleeding, hemodynamic instability, or systemic infection. Renal function, serum electrolytes, and glucose should also be monitored carefully (Hadziyannis, 1997). In-spite of the commonly stated fact that cirrhosis protects from atherosclerosis, yet it was inter- 
esting to notice that it was present in the present cases in both cardiac and vascular field. The least invasive options, such as angioplasty, or minimally invasive revascularization techniques, are likely recommended in patients with advanced cirrhosis. Although there is a role for primary amputation in patients who are poor candidates for surgical revascularization, the morbidity and mortality associated with major amputation remains high (Lowe et al, 1982). At the Wisconsin University, they have used arterial angioplasty as part of their endo-vascular program for treatment of limb threatening ischemia since 2001 (Jude et al, 2001). This treatment option is offered to patients considered high risk or poor candidates for surgical bypass. The main objective in CLI is limb salvage and therefore ambulatory function. Long-term patency comes second after this primary goal. The primary reason for the widespread adoption of endovascular intervention is based on the concept of repeatable recanalization with low complication rates (Kumpe and Rutherford, 1992). In the present protocol, the angiographic study and PTA were performed in the same session, this approach reduces both patient stress and the amount of contrast administered, which are particularly relevant in subjects with cardiac or renal insufficiency (London et al, 1995) The combination of a high technical success rate, a low complications rate and a high limb salvage rate are possible if the PTA physician is particularly skilled and patient care is performed by means of a skilled combined medical and surgical team (Okazaki et al, 2011).

The present protocol employed PTA re-vascularization attempt irrespective of the length, number and localization of obstructions, which led to obtain a high number of technical success and a high limb salvage rate $87.4 \%$ and this was similar to other study that limb salvage was achieved in $96.6 \%$ of patients immediately after the procedure and in $88.3 \%$ a year after the procedure (Ostovan et al. 2011). Also, Zara (2011) achieved nearly the same results. In comparison to Cheng et al. (2003) in spite of insignificant differences of technical success rate, a year primary patency $(56 \%)$ as well as secondary patency (69\%) was significantly lower than in the group $96.6 \%$ of patients immediately after the procedure and in $88.3 \%$ one year after the procedure (Tsetis and Belli, 2004)

\section{Conclusion}

The endovascular management proved to be a good alternative to surgical interference in patients with high surgical as well as anesthetic risk because of their unfitness for general, epidural or spinal anesthesia, intolerability to surgical trauma (decompensation), bleeding tendency (hypoprothrombinaemia and thrombocytopenia), high incidence of wound infections (anergetic \& defective power of wound healing).With recent advances in technology, long and multiple stenotic and occlusive lesions can be treated successfully carrying a lower morbidity and mortality and shorter hospital stay compared with surgery. 
These figures showing the target vessels before and after angioplasty.

Fig. 1: PTA of Iliac artery occlusion

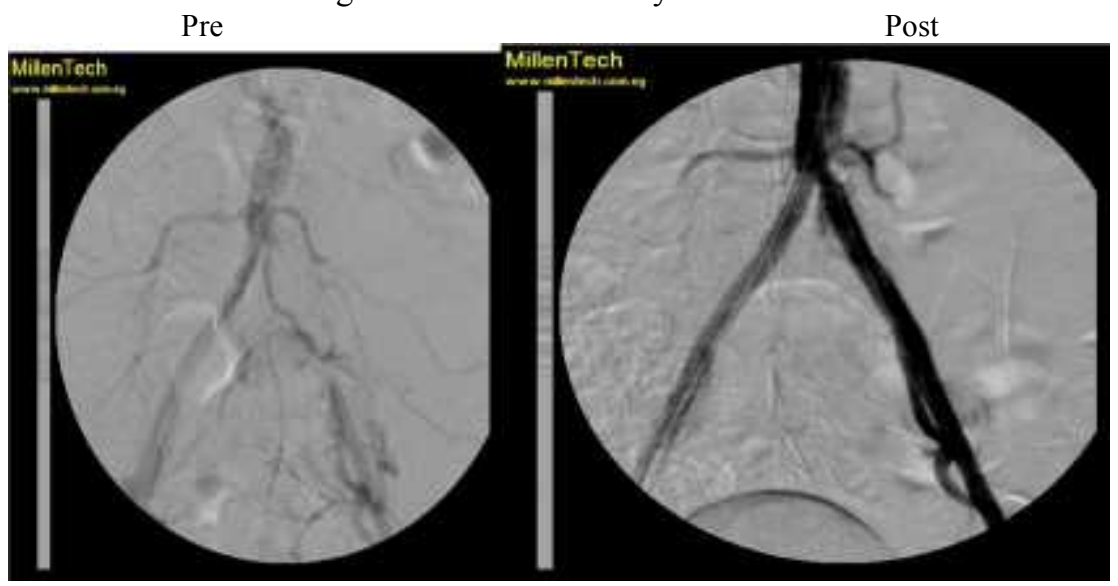

Fig. 2: PTA of superficial femoral artery occlusion Pre
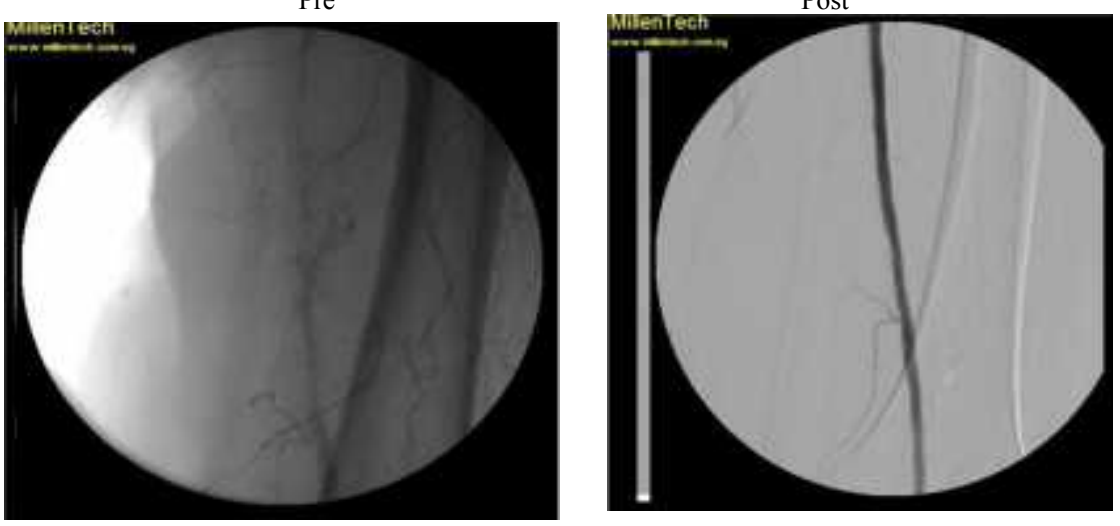

Fig 3: PTA of Popliteal artery occlusion

Pre
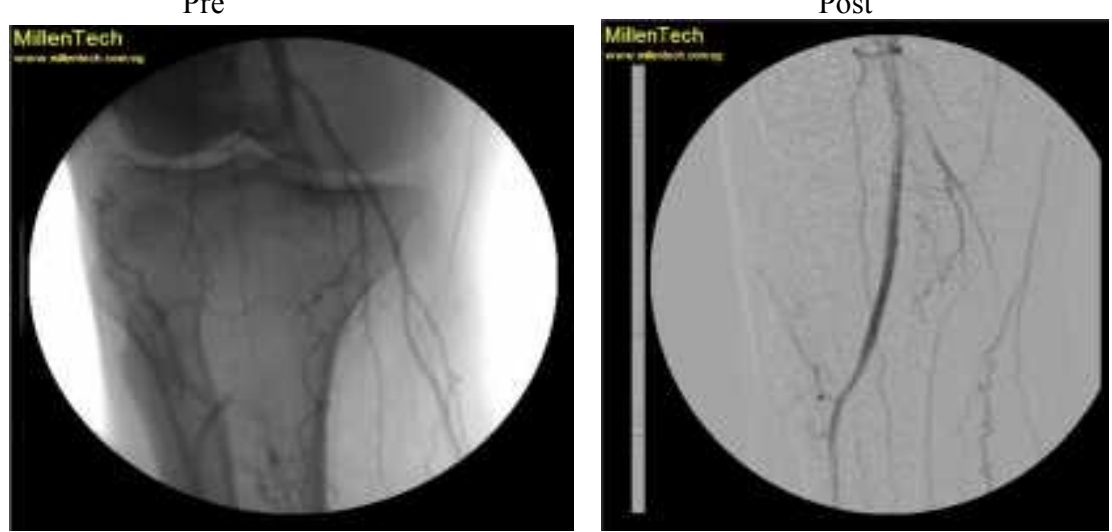


\section{References}

Arora, P, Farid, S, Gannon, MP, Lohr, JW, Dosluoglu, HH, et al, 2013: Low levels of high-density lipoproteins are associated with acute kidney injury following revascularization for chronic limb ischemia. Ren. Fail 35, 6:838-44.

Cheng, SW, Ting, AC, et al, 2003: Angioplasty and primary stenting of high- grade, long-segment superficial femoral artery disease: is it worthwhile? Ann. Vasc. Surg. 17, 4:430-7.

Davies, JN, Ranaboldo, CJ, 1994: Assessment of risk in vascular surgery. Rec. Advan. Surg.17:181-95.

El-Zayadi, AR, Badran, HM, Barakat, MF, Attia, ME, et al, 2005: Hepatocellular carcinoma in Egypt: A single center study over a decade World J. Gastroenterol. 11, 33:5193-8.

Ezzat, S, Abdel-Hamid, M, Eissa, S A, Mokhtar, N, Labib, NA, et al, 2005: Associations of pesticide's, $\mathrm{HCV}, \mathrm{HBV}$, and hepatocellular carcinoma in Egypt. Int. J. Hyg. Environ. Hlth. 208, 5:329-39.

Hadziyannis, SJ, 1997: The spectrum of extrahepatic manifestations in hepatitis $\mathrm{C}$ virus infection. J. Vir. Hepat. 4: 9-28.

Hiatt, WR, Dormandy, JA, Nehler, MR, Harris, KA, Fowkes FG, et al, 2007: TASC II Working Group intersociety consensus for the management of peripheral arterial disease. Eur. J. Vasc. Endovasc. Surg. 33, 1:S1-75.

Jude, EB, Oyibo, SO, Chalmers, N, Boulton, AJ, 2001: Peripheral arterial disease in diabetic and nondiabetic pa- tients: A comparison of severity and outcome. Diabetes Care 24:1433-7.

Kumpe, DA, Rutherford RB, 1992: Percutaneous transluminal angioplasty for lower extremity ischemia. In: Vascular Surgery, Rutheford. RB (ed.), $3^{\text {rd }}$ ed. Philadelphia, PA, USA: W.B. Saunders.

Liao, GS, Wu, MH, Yu, JC, Wu, HS, Chen, TW, et al, 2009: Transection of the esophagus is optional in the Modified Sugiura procedure. Hepatogastroenterology 56, 89:133-8.

London, NJ, Varty, K, Sayers, RD, Thompson, MM, Bell, PR, et al, 1995: Percutaneous transluminal angioplasty for lower-limb critical ischemia. Br. J. Surg. 82:1232-5.

Lowe, GD, Dunlop, DJ, Lawson, DH, et al, 1982: Double-blind controlled clinical trial of ancrod for ischemic rest pain of the leg. Anagiology 33:46-50.

Mohan, P, Colvin, C, Glymph, C, Chandra, RR, Kleiner, DE, et al, 2007: Clinical spectrum and histopathologic features of chronic hepatitis $\mathrm{C}$ infection in children. J. Pediatr. 150, 2: 168-74.

Okazaki, J, Guntani, A, Homma, K, Kyuragi, R, Kawakubo, E, et al, 2011: Mid-term clinical outcome of critical limb ischemia after infrapopliteal bypass surgery. Fukuoka Igaku Zasshi 102, 2:31-9.

Ostovan, MA, Tavassoli, M, Ghofraniha, A, Davaei, MHS, Kojuri, J, 2011: Limb salvage after infra-popliteal angioplasty for critical limb ischemia in surgically high-risk patients in Iran. J. Pak. Med. Stud. 1:385-88. 
Pandey, CK, Karna, ST, Pandey, V $\mathrm{K}$, Tandon, M, Singhal, A, et al, 2012: Perioperative risk factors in patients with liver disease undergoing non-hepatic surgery. World J. Gastrointest. Surg. 4, 12:267-74

Park, KB, Do, YS, Kim, DI, Kim, D K, Kim, YW, et al, 2007: Trans-Atlantic Inter-Society Consensus (TASC) classification system in iliac arterial stent placement: Long-term patency and clinical limitations. J. Vasc. Inter. Radiol. 18, 2:193-201.

Patel, AG, Belgaumkar, AP, James, J, Singh, UP, Carswell, KA, et al, 2011: Video. Single-incision laparoscopic left lateral segmentectomy of colorectal liver metastasis. Surg. Endosc. 25, 2:649-50
Rutherford, IW, 2010: Classification of arterial disease: Vascular Medicine Angiologist.com: Published August 14. Schreuder, TC, Gelderblom, HC, Weegink, CJ, Hamann, D, Reesink, HW, et al, 2008: High incidence of type 1 diabetes mellitus during or shortly after treatment with pegylated interferon alpha for chronic hepatitis $\mathrm{C}$ virus infection. Liver Int. 28, 1:39-46. Tsetis, D, Belli, AM, 2004: The role of infra-popliteal angioplasty Brit. J. Radiology 77:1007-15.

Zara, A, 2011: Infra-popliteal angioplasty versus bypass for critical limb ischemia. JPMS, 1, 3:11-8. 